\title{
Free Vibrations of Timoshenko Beams on Viscoelastic Foundation
}

\author{
Li Peng ${ }^{1, a}$, Ying Wang ${ }^{1, b}$ \\ ${ }^{1}$ College of Civil Engineering, Shanghai Normal University, Shanghai 201418, China \\ apolley28@163.com, bwycyt2000@163.com
}

Keywords: viscoelastic foundation, Timoshenko beam, natural frequency, modal function

Abstract: The transverse free vibration of Timoshenko beams resting on viscoelastic foundation are studied. Complex frequency equations are obtained for pinned-pinned ends. The effects of beam and foundation parameters on natural frequencies are analyzed in numerical examples, and the characteristics of Timoshenko beams are compared with Euler-Bernoulli beams. The results show that natural frequencies grow with increasing the stiffness and the shearing parameters, as well as decreasing the viscoelastic coefficients; the natural frequencies of Timoshenko beams are slightly less than Euler-Bernoulli beams.

\section{Introduction}

Finite beams resting on foundations are widely used in engineering structures, such as roads, airport pavements, and railway engineering equipments. Because of its great practical importance, the vibration analysis of such a system has interested many researchers. Ruge and Birk ${ }^{[1]}$ compared $^{1}$ the Timoshenko and the Euler-Bernoulli beam on Winkler foundation in the frequency-domain. De Rosa $^{[2]}$ numerically solved natural frequencies of Timoshenko beams on the two-parameter foundation. Yokoyama ${ }^{[3]}$ studied the vibration characteristics of a uniform Timoshenko beam-column supported on Pasternak foundation. Malekzadeh ${ }^{[4]}$ analyzed free vibration of Timoshenko beams on elastic foundations. Morfidis ${ }^{[5]}$ investigated vibration of Timoshenko beams on three-parameter elastic foundation.

It is worth noticing that all studies mentioned above are about elastic foundation, without accounting for the effects of damping factor in foundation. Until recent years, researchers began to study vibration of beams on viscoelastic foundation. Most of the published works are focused on dynamic response of such a system subjected to a moving load ${ }^{[6][7]}$ and demonstrated the damping factor is remarkable importance for beams on viscoelastic foundation.

\section{Governing equations}

For a finite elastic Timoshenko beam resting on three-parameter viscoelastic foundation, the governing differential equation of free motion can be written as ${ }^{[8]}$

$$
\begin{aligned}
& \rho A \frac{\partial^{2} w(x, t)}{\partial t^{2}}+k^{\prime} A G\left(\frac{\partial \psi(x, t)}{\partial x}-\frac{\partial^{2} w(x, t)}{\partial x^{2}}\right)+k w(x, t)+\mu \frac{\partial w(x, t)}{\partial t}-G_{\mathrm{P}} \frac{\partial^{2} w(x, t)}{\partial x^{2}}=0 \\
& \rho I \frac{\partial^{2} \psi(x, t)}{\partial t^{2}}-E I \frac{\partial^{2} \psi(x, t)}{\partial x^{2}}+k^{\prime} A G\left(\psi(x, t)-\frac{\partial w(x, t)}{\partial x}\right)=0
\end{aligned}
$$

in which $A, E, G$ and $I$ are cross-sectional area of the beam, the modulus of elasticity, shear modulus of the beam and cross-sectional moment of inertia, and $k^{\prime}, \rho, w$ and $\psi$ are sectional shear coefficient, beam material density, the transverse deflection of the beam and beam slope due to bending. $k, \mu$ and $G_{\mathrm{P}}$ are foundation normal stiffness, damping coefficients and the shear parameter.

Introduce the following dimensionless coordinates or parameters 


$$
w \leftrightarrow \frac{w}{l}, t \leftrightarrow \frac{t}{l} \sqrt{\frac{E}{\rho}}, x \leftrightarrow \frac{x}{l}, k_{1} \leftrightarrow \frac{E}{k^{\prime} G}, k_{2} \leftrightarrow \frac{A l^{2}}{I}, k_{3} \leftrightarrow \frac{k l^{2}}{k^{\prime} A G}, \mu \leftrightarrow \frac{\mu l}{k^{\prime} A G} \sqrt{\frac{E}{\rho}}, k_{4} \leftrightarrow \frac{G_{P}}{k^{\prime} A G}
$$

Substituting Eq.2 into Eq.1 yields the dimensionless equations

$$
\begin{aligned}
& k_{1} w_{{ }_{t t}}+\left(\psi,{ }_{x}-w,{ }_{x x}\right)+k_{3} w+\mu w_{,_{t}}-k_{4} w,_{x x}=0 \\
& k_{1}\left(\psi,{ }_{t t}-\psi,,_{x x}\right)+k_{2}\left(\psi-w,,_{x}\right)=0
\end{aligned}
$$

The vibration solution to Eq.3 can be assumed as

$$
w(x, t)=\sum_{n=1}^{\infty} \zeta_{n}(x) e^{\lambda_{n} t}, \quad \psi(x, t)=\sum_{n=1}^{\infty} \varphi_{n}(x) e^{\lambda_{n} t}
$$

Where $\lambda_{n}=-\delta_{n}+\mathrm{i} \omega_{n},(n=1,2,3 \ldots), \delta_{n}$ is the $n$th decrement coefficient, $\omega_{n}$ is the $n$th natural frequency.

Substituting Eq.4 into Eq.3 yields

$$
\begin{aligned}
& \left(\lambda_{n}{ }^{2} k_{1}+k_{3}+\lambda_{n} \mu\right) \zeta_{n}(x)-\left(1+k_{4}\right) \zeta_{n}^{\prime \prime}(x)+\varphi_{n}^{\prime}(x)=0 \\
& \left(\lambda_{n}{ }^{2} k_{1}+k_{2}\right) \varphi_{n}(x)-k_{2} \zeta_{n}^{\prime}(x)-k_{1} \varphi_{n}^{\prime \prime}(x)=0
\end{aligned}
$$

Eliminating $\zeta_{n}(x)$ or $\varphi_{n}(x)$ from Eq.5, one can get

$$
\begin{aligned}
& a \zeta_{n}^{(4)}(x)+b \zeta_{n}^{\prime \prime}(x)+c \zeta_{n}(x)=0 \\
& a \varphi_{n}^{(4)}(x)+b \varphi_{n}^{\prime \prime}(x)+c \varphi_{n}(x)=0
\end{aligned}
$$

where

$$
\begin{aligned}
& a=k_{1}\left(1+k_{4}\right) \\
& b=-k_{1} k_{3}-k_{2} k_{4}-\mu k_{1} \lambda_{n}-\left(1+k_{1}+k_{4}\right) k_{1} \lambda_{n}{ }^{2} \\
& c=k_{2} k_{3}+\mu k_{2} \lambda_{n}+k_{1}\left(k_{2}+k_{3}\right) \lambda_{n}{ }^{2}+\mu k_{1} \lambda_{n}{ }^{3}+k_{1}{ }^{2} \lambda_{n}{ }^{4}
\end{aligned}
$$

The general solutions of Eq. 6 are

$$
\begin{aligned}
& \zeta_{n}(x)=C_{n 1}\left(e^{\gamma_{n 1} x}+C_{n 2} e^{\gamma_{n 2} x}+C_{n 3} e^{\gamma_{n 3} x}+C_{n 4} e^{\gamma_{n 4} x}\right) \\
& \varphi_{n}(x)=D_{n 1}\left(e^{\gamma_{n 1} x}+D_{n 2} e^{\gamma_{n 2} x}+D_{n 3} e^{\gamma_{n 3} x}+D_{n 4} e^{\gamma_{n 4} x}\right)
\end{aligned}
$$

Substituting Eq.4 and Eq.8 into Eq.3 yields

$$
\begin{gathered}
D_{n 1}=-\frac{1}{\gamma_{n 1}}\left[\left(\lambda_{n}^{2} k_{1}+k_{3}+\lambda_{n} \mu\right)-\left(1+k_{4}\right) \gamma_{n 1}^{2}\right] C_{n 1} \\
D_{n j}=\frac{\gamma_{n 1}}{\gamma_{n j}} \frac{\left[\left(\lambda_{n}^{2} k_{1}+k_{3}+\lambda_{n} \mu\right)-\left(1+k_{4}\right) \gamma_{n j}{ }^{2}\right]}{\left[\left(\lambda_{n}{ }^{2} k_{1}+k_{3}+\lambda_{n} \mu\right)-\left(1+k_{4}\right) \gamma_{n 1}{ }^{2}\right]} C_{n j}
\end{gathered}
$$

Based on Eq.4,under the dimensionless pinned-pinned boundary conditions, one can get

$$
\zeta_{n}(0)=\zeta_{n}(1)=0, \quad \varphi_{n}^{\prime}(0)=\varphi_{n}^{\prime}(1)=0
$$

Substitution of Eq.8 and Eq.9 into Eq.10 yields

$$
\left(\begin{array}{cccc}
1 & 1 & 1 & 1 \\
e^{\gamma_{n 1}} & e^{\gamma_{n 2}} & e^{\gamma_{n 3}} & e^{\gamma_{n 4}} \\
B_{n 1} & B_{n 2} & B_{n 3} & B_{n 4} \\
B_{n 1} e^{\gamma_{n 1}} & B_{n 2} e^{\gamma_{n 2}} & B_{n 3} e^{\gamma_{n 3}} & B_{n 4} e^{\gamma_{n 4}}
\end{array}\right)\left(\begin{array}{c}
1 \\
C_{n 2} \\
C_{n 3} \\
C_{n 4}
\end{array}\right) C_{n 1}=\left(\begin{array}{l}
0 \\
0 \\
0 \\
0
\end{array}\right)
$$

where

$$
B_{n j}=-\left(\lambda_{n}{ }^{2} k_{1}+k_{3}+\lambda_{n} \mu\right)+\left(1+k_{4}\right) \gamma_{n j}{ }^{2}
$$


For the nonzero solution condition of Eq.11, determinant of the coefficient matrix must be zero

$$
\begin{aligned}
& \left(e^{\gamma_{n 2}}-e^{\gamma_{n 3}}\right)\left(e^{\gamma_{n 1}}-e^{\gamma_{n 4}}\right)\left(B_{n 2} B_{n 3}+B_{n 1} B_{n 4}\right)+\left(e^{\gamma_{n 1}}-e^{\gamma_{n 3}}\right)\left(e^{\gamma_{n 2}}-e^{\gamma_{n 4}}\right) \\
& \left(-B_{n 1} B_{n 3}-B_{n 2} B_{n 4}\right)+\left(e^{\gamma_{n 1}}-e^{\gamma_{n 2}}\right)\left(e^{\gamma_{n 3}}-e^{\gamma_{n 4}}\right)\left(B_{n 1} B_{n 2}+B_{n 3} B_{n 4}\right)=0
\end{aligned}
$$

Based on Eq.6 and Eq.13, transcendental equation about $\lambda_{n}$ that can be numerically solved. As real and imaginary parts of $\lambda_{n}$, natural frequency $\omega_{n}$ and decrement coefficient $\delta_{n}$ can also be numerically calculated.

\section{Numerical Results}

Consider the modelling of the uncertainty of track as a finite elastic Timoshenko beam, the parameters of the beam and foundation come from the model beam ${ }^{[8]}$.

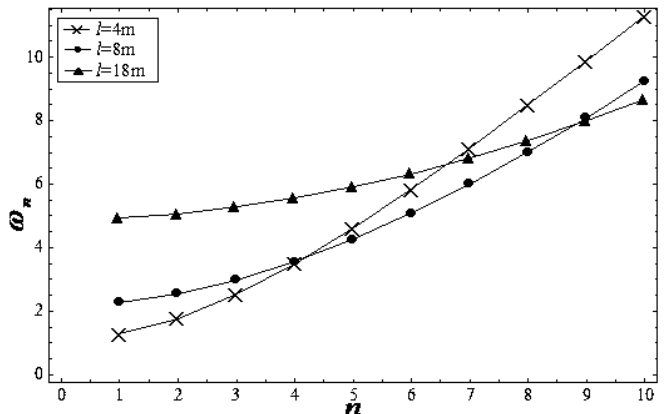

(a) length of beam

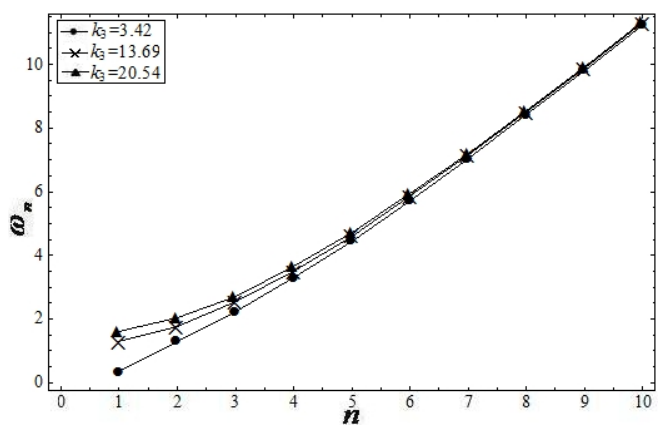

(c) foundation stiffness parameters

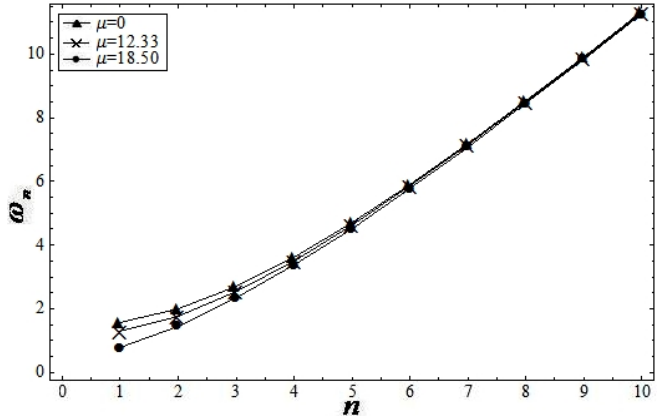

(b) viscous damping coefficients

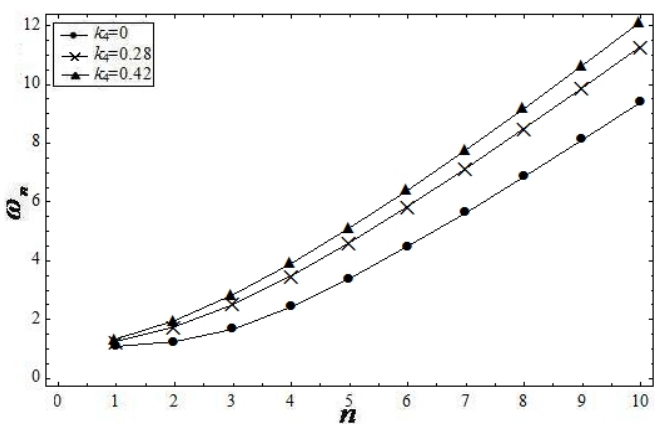

(d) shear parameters of foundation

Fig. 1. First ten natural frequencies

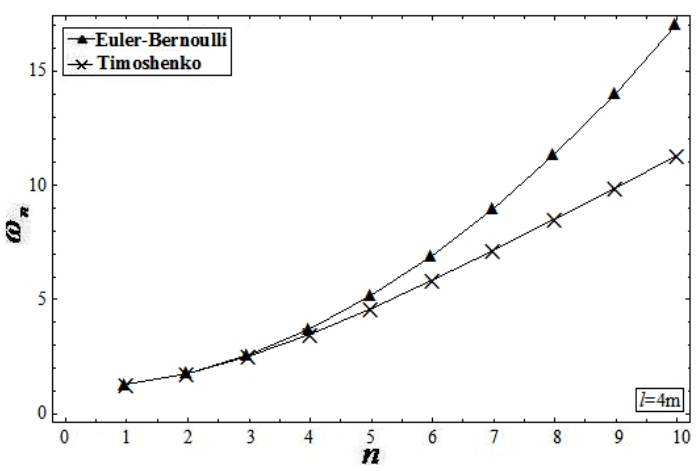

(a) $\quad l=\mathbf{4 m}$

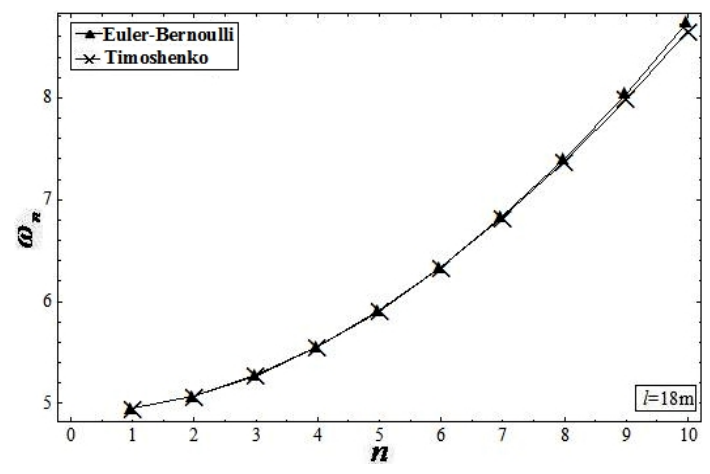

(b) $\quad l=18 \mathrm{~m}$

Fig. 2. First ten natural frequencies of Timoshenko beams and Euler-Bernoull beams

Fig.1shows the low order natural frequencies of shorter beam are less than that of longer beam, but the high order natural frequencies of shorter beam grows quickly and more than that of longer 
beam. With the foundation viscous damping coefficients increasing, the natural frequencies decrease, and the difference between low order natural frequencies is greater than that of high order. With the growth of the stiffness and the shear parameters, the natural frequencies increase. For foundation stiffness parameters, the difference of the low order natural frequencies is larger than that of high orders. But for different shear parameters, the natural frequencies of first order are almost same, the natural frequencies of the greater foundation shear parameters increase more quickly with the orders growing.

In Fig.2, resting on the same foundation, the natural frequency values of Timoshenko beams are lower than that of Euler-Bernoulli beams, but the difference between them decrease with the length of the beams increasing.

\section{Conclusions}

In this paper, the free transverse vibration of Timoshenko beams on three-parameter viscoelastic foundation is investigated. Under the simple boundary condition, the formulae for natural frequencies of the free transverse vibration are developed. The numerical examples show that, with order growing, the natural frequencies increase, and the decrement coefficients slightly decrease. The natural frequencies increase with the growth of beam length, stiffness and shear parameters of the foundation, and the decreasing of viscous damping coefficients. For foundation stiffness parameters, the differences between the low order natural frequencies are greater than that between high orders. The high order natural frequencies with the larger foundation shear parameters increase more quickly. Moreover, on the same foundation, the natural frequency values of Timoshenko beams are lower than that of Euler-Bernoulli beams.

\section{References}

[1] P. Ruge and C. Birk: 'A comparison of infinite Timoshenko and Euler-Bernoulli beam models on Winkler foundation in the frequency- and time-domain', Journal of Sound and Vibration, 2007, 304, 932-947.

[2] M. A. De Rosa: 'Free vibrations of Timoshenko beams on two-parameter elastic foundation', Computers \& Structures, 1995, 57(1), 151-156.

[3] T. Yokoyama: 'Vibration analysis of Timoshenko beam-Columns on two-parameter elastic foundations', Computers \& Structures, 1996, 61(6), 995-1007.

[4] P. Malekzadeh and G. Karami: 'DQEM for free vibration analysis of Timoshenko beams on elastic foundations', Computational Mechanics, 2003, 31, 219-228.

[5] K. Morfidis: 'Vibration of Timoshenko beams on three-parameter elastic foundation', Computers \& Structures, 2010, 88, 294-308.

[6] F. F. ÇalIm: 'Dynamic analysis of beams on viscoelastic foundation', European Journal of Mechanics A/Solids, 2009, 28, 469-476.

[7] H. Ding, L. Q. Chen, and S. P. Yang: 'Convergence of Galerkin truncation for dynamic response of finite beams on nonlinear foundations under a moving load', Journal of Sound and Vibration, 2012, 331, 2426-2442.

[8] D. Younesian, M. H. Kargarnovin, D. J. Thompson, and C. J. C. Jones: 'Parametrically excited vibration of a Timoshenko beam on random viscoelastic foundation subjected to a harmonic moving load', Nonlinear Dynamics, 2005, 45, 75-93. 